\title{
The right hippocampus participates in short-term memory maintenance of object-location associations
}

\author{
Carinne Piekema, ${ }^{\mathrm{a}, \mathrm{b}, *}$ Roy P.C. Kessels, ${ }^{\mathrm{a}, \mathrm{c}}$ Rogier B. Mars, ${ }^{\mathrm{b}, \mathrm{d}}$ \\ Karl Magnus Petersson, ${ }^{\mathrm{b}}$ and Guillén Fernández ${ }^{\mathrm{b}, \mathrm{e}}$ \\ ${ }^{a}$ Helmholtz Instituut, Department of Psychonomics, Utrecht University, Heidelberglaan 2, 3584 CS Utrecht, The Netherlands \\ ${ }^{\mathrm{b}}$ F.C. Donders Centre for Cognitive Neuroimaging at the Radboud University Nijmegen, P.O. Box 9101, 6500 HB Nijmegen, The Netherlands \\ ${ }^{\mathrm{c}}$ Department of Neurology, University Medical Center Utrecht, Heidelberglaan 100, 3584 CX Utrecht, The Netherlands \\ ${ }^{\mathrm{d}}$ Nijmegen Institute for Cognition and Information, Radboud University Nijmegen, P.O. Box 9104, 6500 HE Nijmegen, The Netherlands \\ ${ }^{\mathrm{e}}$ Department of Neurology, Radboud University Nijmegen Medical Centre, P.O. Box 9101, 6500 HB Nijmegen, The Netherlands
}

Received 28 February 2006; revised 19 June 2006; accepted 20 June 2006

Available online 10 August 2006

Doubts have been cast on the strict dissociation between short- and long-term memory systems. Specifically, several neuroimaging studies have shown that the medial temporal lobe, a region almost invariably associated with long-term memory, is involved in active short-term memory maintenance. Furthermore, a recent study in hippocampally lesioned patients has shown that the hippocampus is critically involved in associating objects and their locations, even when the delay period lasts only $8 \mathrm{~s}$. However, the critical feature that causes the medial temporal lobe, and in particular the hippocampus, to participate in active maintenance is still unknown. This study was designed in order to explore hippocampal involvement in active maintenance of spatial and non-spatial associations. Eighteen participants performed a delayed-match-to-sample task in which they had to maintain either object-location associations, color-number association, single colors, or single locations. Whole-brain activity was measured using eventrelated functional magnetic resonance imaging and analyzed using a random effects model. Right lateralized hippocampal activity was evident when participants had to maintain object-location associations, but not when they had to maintain object-color associations or single items. The present results suggest a hippocampal involvement in active maintenance when feature combinations that include spatial information have to be maintained online.

(C) 2006 Elsevier Inc. All rights reserved.

Keywords: Associative memory; fMRI; Spatial; Working memory; Longterm memory

\footnotetext{
* Corresponding author. F.C. Donders Centre for Cognitive Neuroimaging, P.O. Box 9101, 6500 HB Nijmegen, The Netherlands.

E-mail address: Carinne.Piekema@fcdonders.ru.nl (C. Piekema).

Available online on ScienceDirect (www.sciencedirect.com).
}

\section{Introduction}

Double dissociations between distinct types of memory have led to the definition of separate memory systems, i.e., specific neural networks that support specific mnemonic processes (Squire et al., 1993; Schacter and Tulving, 1994; for a review, see Gabrieli, 1998). Importantly, this research has suggested a dissociation between short- and long-term memory systems. Patients with bilateral medial temporal lobe lesions typically exhibit normal short-term memory performance, but severely diminished consciously accessible longterm memory (i.e., episodic or declarative memory) (Scoville and Milner, 1957); conversely, patients with perisylvian lesions show impaired verbal short-term memory performance, but normal longterm memory for verbal information (Shallice and Warrington, 1970; see also Vallar and Papagno, 1995). Persistent episodic memories are thought to be based on changes in neural structures, which are stable and do not require active maintenance by sustained neural firing (Bliss and Lomo, 1973; Engert and Bonhoeffer, 1999; Shors et al., 2001). In contrast, short-term memories are based on sustained neural firing patterns in brain regions representing, for instance, the perceptual or verbal features of a memory. This firing pattern is actively maintained during the delay when information is kept in short-term memory, typically spanning time periods of up to several seconds (Fuster and Alexander, 1971; Goldman-Rakic, 1987; Miller et al., 1996; Miyashita and Chang, 1998).

Lately however, doubts have been cast on this strict dissociation between short- and long-term memory (e.g., Wagner, 1999; Davachi et al., 2001; Ranganath and Blumenfeld, 2005; Olson et al., 2006). Several electrophysiological studies in non-human primates and functional neuroimaging studies in humans revealed delay period activity in the medial temporal lobe, a structure traditionally implicated solely in long-term memory, related to active maintenance of short-term or working memory (e.g., Watanabe and Niki, 1985; Cahusac et al., 1993; 
Miller et al., 1993; Suzuki et al., 1997; Mitchell et al., 2000a; Stern et al., 2001; Ranganath and D'Esposito, 2001; Cabeza et al., 2002; Schon et al., 2004; Ranganath et al., 2005a). Furthermore, recently lesion evidence has become available, indicating that amnesic patients with bilateral hippocampal lesions cannot maintain object-location associations over short time periods of a few seconds (Olson et al., 2006). To accommodate the new findings, models suggesting an interaction between the two types of memory have recently been proposed (e.g., Baddeley, 2000; Jensen and Lisman, 2005).

Although the functional role of sustained activity in the medial temporal lobe, and specifically in the hippocampus, remains elusive, a recent proposal for an additional working memory module termed the 'episodic buffer' might characterize its function (Baddeley, 2000). This buffer is proposed to be a limited capacity system that provides, through active maintenance, a temporary storage of information in a multimodal code capable of associating information from subsidiary unimodal systems and long-term memory into a coherent representation. Hence, in binding together related information, sustained hippocampal activity might be important for both working memory maintenance of associative information and the encoding of new declarative memories (Schon et al., 2004; Ranganath et al., 2005a).

Complicating this issue even further, it remains unclear under which precise circumstances the hippocampus shows sustained activity during a delay period of a short-term memory task. Both animal and human studies have shown that the hippocampus is critically important for associative memory tasks in which different non-related components have to be associated. In those tasks, the hippocampus functions as an integrative structure, binding together the different aspects of an experience. However, there is some debate on whether the hippocampal contribution to associative memory is related mainly to spatial associations (O'Keefe and Nadel, 1978; Chalfonte and Johnson, 1996; Wood et al., 2004) or to associations in general (Eichenbaum et al., 1994; Henke et al., 1997, 1999; Brasted et al., 2003; Luo and Niki, 2005; for a review, see Kessels et al., 2001). Given this debate, one may ask whether sustained hippocampal activity occurs during active maintenance of spatial associations only or associations in general. To tackle this issue, we conducted an event-related fMRI study, probing delay period activity in a delayed-match-to-sample task (Sternberg, 1966) in which the participants had to maintain either spatial or non-spatial associations.

\section{Materials and methods}

\section{Participants}

Eighteen right-handed (self report) healthy university students (10 males, mean age 25.2 years, range $20-32$ years, $1-5$ years of university level education) with normal or corrected-to-normal vision participated in the experiment. All participants provided written informed consent according to institutional guidelines of the local ethics committee (CMO region Arnhem-Nijmegen, Netherlands) and the declaration of Helsinki.

\section{Experimental setup}

During scanning, participants lay comfortably in a supine position in the MR scanner. An adjusted padded head holder restricted any head movements. Visual stimuli were projected onto a translucent screen at the back of the scanner and seen by the participants through a mirror in front of their eyes. All stimuli were presented on a black background. Button press responses were recorded via an MR-compatible keypad.

Participants performed a 3-item delayed-match-to-sample task (Sternberg, 1966) with eight different one-digit numbers (1-8; visual angle $\sim 3^{\circ}$ ) as stimuli, presented randomly in eight different colors (green, yellow, orange, red, pink, blue, cyan, and gray) and at eight different locations on the screen. Stimuli were presented at the corners of a horizontally and vertically centered, symmetric, invisible octogram $\left(\sim 18^{\circ}\right.$ off center $)$. We used a $2 \times 2$ factorial design with the factors ASSOCIATION (single vs. multiple) and SPACE (location vs. color). Thus, the experiment included four conditions in which the participants were instructed to retain (a) the location at which the stimuli were presented (spatial single), (b) the color of the stimuli (non-spatial single), (c) the combination of the identity of the stimuli and their location (spatial multiple), or (d) the combination of the identity of the stimuli and their color (nonspatial multiple).

An instructive cue presented for $1 \mathrm{~s}$ at the start of each trial indicated which feature or features had to be retained. Subsequently, three items were presented sequentially ( $1 \mathrm{~s}$ per stimulus). After a variable delay (range: 9-20 s, uniform distribution), during which the participant was instructed to fixate on a centrally presented cross, one probe item was presented (2 s; see Fig. 1). Participants were instructed to respond to the probe by a button press with the right index finger if the feature or specific combination of features specified by the initial cue belonged to the current set, or with the right middle finger if this did not belong to the current set. Trials were separated by a variable inter-trial interval (range: 4-9 s, uniform distribution).

This setup allowed us to probe sustained blood oxygen level dependent (BOLD) responses associated with the delay interval. Introducing a mismatch between the start of successive trials and volume acquisition allowed us to characterize the evoked hemodynamic response at a finer temporal resolution than the actual TR (Josephs et al., 1997). The variable delay intervals allowed us to partition the variance into separate components for the stimulus set, the delay interval, and the probe and subsequent response (see Toni et al., 1999 for a similar approach). Eighty-eight trials from four conditions (22 trials per condition, $50 \%$ matches) were presented randomly intermixed over the course of the scanning session. Before the experiment, simulations were run to determine the optimal distribution of trial types and delay lengths as to ensure sufficiently low

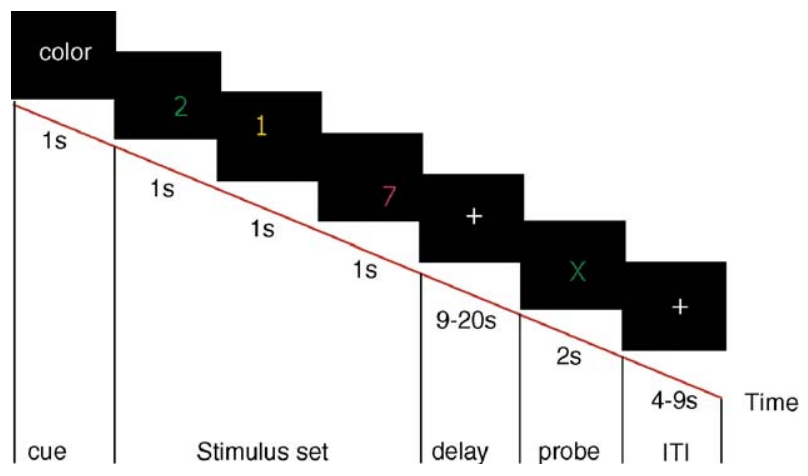

Fig. 1. Experimental design. 
correlations to allow each of the separated regressors to be estimated reliably (Dale, 1999). A unique stimulus list was created for each participant, randomizing number, color, location, and delay length.

Prior to the start of the experiment and in order to account for initial learning effects, participants practiced the task for 32 trials outside the scanner. The practice material was different from the stimulus set used in the actual scanning session. Subjects were instructed to prioritize correct responding over speed.

\section{fMRI data acquisition}

During MRI scanning, whole head T2*-weighted EPI-BOLD fMRI data were acquired within one run of approximately 998 volumes with a Siemens Sonata 1.5 T MR scanner using an ascending slice acquisition sequence (33 axial slices, volume $\mathrm{TR}=2.29 \mathrm{~s}, \mathrm{TE}=30 \mathrm{~ms}, 90^{\circ}$ flip-angle, slice-matrix size $=64 \times 64$, slice thickness $=3.0 \mathrm{~mm}$, slice gap $=0.5 \mathrm{~mm}$, field of view $=224 \mathrm{~mm}$ resulting in an in-plane resolution of $3.5 \times 3.5 \mathrm{~mm}$ ). Following the experimental session, a high-resolution structural image was acquired, using a T1-weighted MP-RAGE sequence (volume $\mathrm{TR}=2250 \mathrm{~ms}, \mathrm{TE}=3.93 \mathrm{~ms}, 15^{\circ}$ flip-angle, 176 sagittal slices, slice-matrix size $=256 \times 256$, slice thickness $=1 \mathrm{~mm}$, slice gap $=0 \mathrm{~mm}$, field of view $=256 \mathrm{~mm}$ ).

\section{Behavioral data analysis}

The error rates per condition, acquired during scanning, were subjected to a repeated measures analysis of variance with the factors ASSOCIATION (single versus multiple) and SPACE (location versus color). Post hoc, significant interactions were investigated using paired-samples $t$ tests.

\section{fMRI data analysis}

Image pre-processing and statistical analysis were performed using the SPM2 software (http://www.fil.ion.ucl.ac.uk/spm). The first five volumes of each participant's data set were discarded to allow for T1 equilibration. Each subject's functional images were spatially realigned using a sinc interpolation algorithm that estimates rigid body transformations (translations, rotations) by minimizing head movements between each image and the reference image (Friston et al., 1995b). The subject's structural MR image was coregistered to the mean of the functional images, using mutual information optimization. Subsequently, the functional images were slice-time-corrected, spatially normalized, and resampled to create $2 \mathrm{~mm}$ isotropic voxels, and transformed into a common stereotactic space, as defined by the SPM2 MNI T1 template, and spatially filtered by convolving the functional images with an isotropic 3D Gaussian kernel (10 mm FWHM). The choice of smoothing kernel was optimized for the hippocampus, our primary region of interest for this study, as suggested by a comparative study (Hopfinger et al., 2000). The fMRI data were statistically analyzed using the general linear model and statistical parametric mapping (Friston et al., 1995a). For each of the four conditions, separate regressors were created for cue and stimulus set presentation, delay interval, and probe and response-related effects. Separate regressors were created for correct and incorrect trials. In addition, the realignment parameters were included in the model to account for movement-related variability. The data were highpass-filtered $(128 \mathrm{~s})$ to account for various low-frequency effects. Temporal autocorrelation was modeled as an AR(1) process.

Tackling the question at issue, we created contrast images of delay-related effects on correct trials only and entered them into a second-level analysis, treating subjects as a random variable. Hence, we are probing brain activity related to sustained activity of short-term memory.

At the second level, the data were modeled as an ANOVA with the factors ASSOCIATON and SPACE with non-sphericity correction for correlated repeated measures. As a first explorative analysis, we reviewed the main effects and interactions over the whole brain using a threshold of $p<0.001$ (uncorrected). Following this preliminary inspection, we further employed cluster size statistics as the test statistic for our whole-brain analyses, only considering clusters of activation significant at a threshold of $p \leq 0.05$ (corrected for multiple comparisons). The atlas of Duvernoy et al. (1991) was used to identify relevant anatomical landmarks.

Given our regional specific hypothesis regarding the hippocampus, we took a slightly different hierarchical approach for this region (Friston et al., 1996). Activation in the hippocampus during the preliminary whole-brain analysis at $p<0.001$ uncorrected was taken as a justification for an a priori defined region of interest analysis (ROI). The hippocampal region of interest was defined using the WFU PickAtlas Toolbox for SPM (Maldjian et al., 2003). Both the cluster size and local maximum test statistics were employed in this ROI

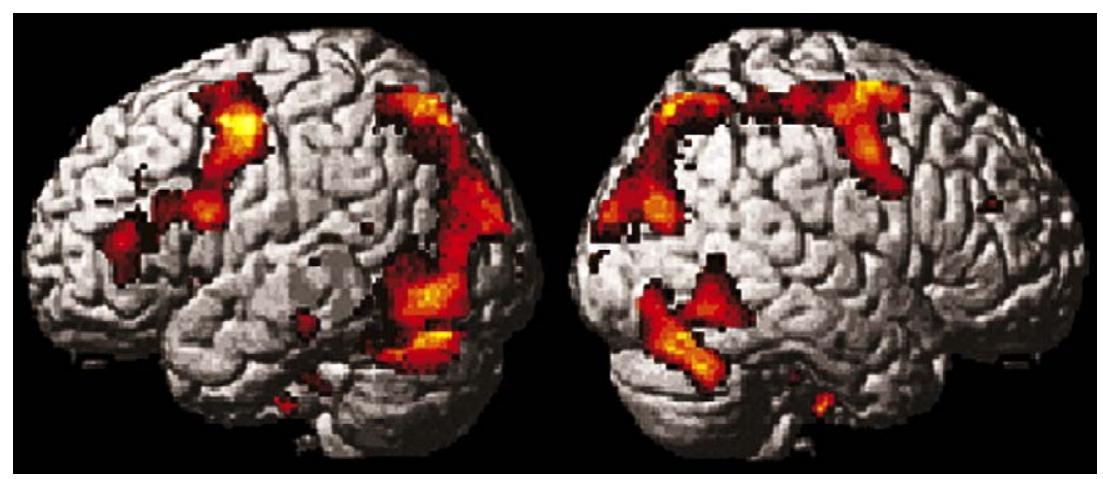

Fig. 2. Delay period activation of all four conditions against a low-level visual fixation baseline rendered onto the surface of a canonical brain. 
analysis and all reported $p$ values are corrected for multiple non-independent comparisons based on the family-wise error correction (Friston et al., 1996) (Fig. 2).

\section{Results}

\section{Behavioral data}

Participants performed significantly above chance level $(50 \%)$ in all conditions (1) 'spatial single': mean correct $87 \%$ (SD 10\%; mean RT: $1533 \mathrm{~ms}$ ), $t_{(17)}=16.0, p<0.0001$, (2) 'nonspatial single': mean correct 93\% (SD 6\%; mean RT: $1488 \mathrm{~ms}$ ), $t_{(17)}=31.3, p<0.0001$, (3) 'spatial multiple': mean correct $91 \%$ (SD 7\%; mean RT: $1720 \mathrm{~ms}$ ), $t_{(17)}=24.3 p<0.0001$, and (4) 'non-spatial multiple': mean correct $78 \%$ (SD 14\%; mean RT: $1999 \mathrm{~ms}), t_{(17)}=8.1, p<0.0001$. These behavioral results are consistent with earlier studies using a similar task (e.g., Mitchell et al., 2000a,b). Recognition performance varied significantly across conditions. There was a main effect of ASSOCIATION (single vs. multiple: $F_{(1,17)}=9.1, p<0.01$ ) and an interaction between the factors ASSOCIATION and SPACE $F_{(1,17)}=25.7$, $p<0.0001$. A post hoc $t$ test indicated that participants performed significantly better in the 'spatial multiple' condition compared to the 'non-spatial multiple' condition $t_{(17)}=4.3$, $p<0.001$. In addition, subjects performed better on the 'nonspatial single' condition compared to the 'spatial single' condition $t_{(17)}=3.0, p<0.01$.

\section{fMRI data}

Whole-brain analyses showed that a variety of regions commonly associated with active working memory maintenance (i.e., specific parietal, temporal, and frontal regions; for a review, see Wager and Smith, 2003) were active when all delay periods on correct trials (independent of condition) were compared to a low-level inter-trial visual fixation baseline (see Table 1). This finding shows that our setup was suitable to assess the brain regions involved in short-term memory maintenance.

Our aim was to reveal critical features that cause medial temporal participation in the delay period of a short-term memory task, focusing on spatial binding and binding in general. Hence, we performed an ANOVA with the factors of ASSOCIATION and SPACE on the correct trials only. In the medial temporal lobe, this ANOVA revealed an interaction in the right hippocampus $(p<0.001$ uncorrected). Given our regional specific hypothesis, we investigated the hippocampal cluster using a hippocampal mask defined by the WFU PickAtlas in SPM (Maldjian et al., 2003). This revealed a cluster with a local maximum at $[30,-20,-10]\left(t_{(51)}=4.08\right.$, $p=0.033$ corrected) which was most active in the spatial multiple condition. To confirm that the hippocampus was indeed activated during the delay period when number-locations associations were maintained, we contrasted activity in the spatial multiple condition with activation during baseline and found indeed a hippocampal cluster with a local maximum at $[30-24-12]\left(t_{(51)}=3.17, p=0.015\right.$ corrected $)$. Moreover, the interaction blob remains when inclusively masked with the spatial multiple> baseline contrast. All other conditions did not activate the hippocampus significantly when contrasted to the baseline (see Fig. 3b). To ensure that the observed activation was indeed localized in the hippocampus, we increased the
Table 1

Regions showing delay-related activation (correct trials only) against a lowlevel visual fixation baseline

\begin{tabular}{|c|c|c|c|c|c|}
\hline General delay effects & $\begin{array}{l}\text { Brodmann's } \\
\text { area }\end{array}$ & $X$ & $Y$ & $Z$ & $\begin{array}{l}t \\
\text { value }\end{array}$ \\
\hline \multirow[t]{2}{*}{ Left anterior cingulate cortex } & 32 & -6 & 12 & 60 & 6.06 \\
\hline & 32 & -10 & 18 & 46 & 4.59 \\
\hline \multirow[t]{2}{*}{ Left superior frontal gyrus } & 8 & -10 & 20 & 50 & 4.48 \\
\hline & 8 & -12 & 20 & 54 & 4.19 \\
\hline \multirow{4}{*}{$\begin{array}{l}\text { Right middle/superior frontal } \\
\text { gyrus }\end{array}$} & $6 / 8$ & 24 & -8 & 48 & 6.92 \\
\hline & 6 & 50 & -2 & 40 & 6.59 \\
\hline & 6 & 26 & -2 & 60 & 7.18 \\
\hline & $6 / 8$ & 20 & 8 & 60 & 7.18 \\
\hline \multirow{3}{*}{$\begin{array}{l}\text { Left middle/superior frontal } \\
\text { gyrus }\end{array}$} & $6 / 8$ & -28 & -2 & 48 & 8.04 \\
\hline & 6 & -50 & -4 & 50 & 7.66 \\
\hline & 6 & -24 & 0 & 60 & 5.96 \\
\hline \multirow{2}{*}{$\begin{array}{l}\text { Right inferior/middle frontal } \\
\text { gyrus }\end{array}$} & $6 / 44$ & 36 & -4 & 30 & 4.06 \\
\hline & $6 / 44$ & 40 & 4 & 28 & 3.84 \\
\hline Right orbitofrontal cortex & 11 & 20 & 28 & -8 & 4.78 \\
\hline Right precentral gyrus & $4 / 6$ & 36 & -22 & 54 & 4.81 \\
\hline Left precentral gyrus & $4 / 6$ & -42 & -14 & 42 & 3.89 \\
\hline \multirow[t]{2}{*}{ Right precuneus } & 7 & 6 & -46 & 62 & 8.71 \\
\hline & 7 & 8 & -52 & 60 & 6.83 \\
\hline \multirow[t]{2}{*}{ Left precuneus } & 7 & -10 & -50 & 60 & 6.71 \\
\hline & 7 & -14 & -52 & 60 & 6.67 \\
\hline Right superior parietal lobule & 7 & 18 & -52 & 54 & 8.28 \\
\hline Left superior parietal lobule & 7 & -20 & -66 & 54 & 9.19 \\
\hline $\begin{array}{l}\text { Right superior/inferior parietal } \\
\text { lobule }\end{array}$ & $7 / 40$ & -28 & -70 & 34 & 5.89 \\
\hline $\begin{array}{l}\text { Left superior/inferior parietal } \\
\text { lobule }\end{array}$ & $7 / 40$ & -24 & -60 & 46 & 6.88 \\
\hline Right inferior occipital gyrus & $17 / 18$ & 8 & -92 & 2 & 6.44 \\
\hline \multirow[t]{2}{*}{ Left inferior occipital gyrus } & $18 / 19$ & -42 & -72 & -8 & 6.45 \\
\hline & $18 / 19$ & -50 & -64 & -12 & 6.44 \\
\hline ital gyrus & $18 / 19$ & 14 & -92 & 18 & 6.32 \\
\hline Left middle occipital gyrus & $18 / 19$ & -16 & -92 & 20 & 7.25 \\
\hline Right superior occipital gyrus & 19 & 26 & -82 & 26 & 5.17 \\
\hline Left superior occipital gyrus & 19 & -26 & -80 & 30 & 6.23 \\
\hline \multirow{2}{*}{$\begin{array}{l}\text { Right inferior occipital/temporal } \\
\text { gyrus }\end{array}$} & $19 / 20 / 37$ & 48 & -60 & -10 & 4.67 \\
\hline & $19 / 20 / 37$ & 52 & -50 & -12 & 3.94 \\
\hline $\begin{array}{l}\text { Left inferior occipital/temporal } \\
\text { gyrus }\end{array}$ & $19 / 20 / 37$ & -46 & -66 & -10 & 6.40 \\
\hline Right lingual/fusiform gyrus & $18 / 19$ & 20 & -72 & -6 & 8.40 \\
\hline Left lingual/fusiform gyrus & $18 / 19$ & -14 & -74 & -4 & 8.87 \\
\hline Left fusiform gyrus & $19 / 37$ & -38 & -60 & -10 & 6.11 \\
\hline $\begin{array}{l}\text { Left fusiform/parahippocampal } \\
\text { gyrus }\end{array}$ & $19 / 32$ & -32 & -46 & -22 & 9.33 \\
\hline \multirow[t]{2}{*}{ Right putamen/globus pallidus } & & 24 & 8 & 10 & 4.34 \\
\hline & & 24 & -12 & 10 & 4.31 \\
\hline \multirow[t]{2}{*}{ Left putamen/globus pallidus } & & -22 & 6 & 12 & 5.91 \\
\hline & & -20 & -8 & 12 & 4.65 \\
\hline
\end{tabular}

All local maxima listed belong to clusters significant at $p \leq 0.05$ (corrected).

localization accuracy by using a smoothing kernel of $6 \mathrm{~mm}$ FWHM (Fig. 3d).

No significant interaction was found in the left medial temporal lobe. We explicitly tested this hemispheric difference explicitly by comparing the right and the left hippocampal activation levels. We tested for the nearest suprathreshold cluster in the right vs. left comparison and observed a significant difference (cluster $p<0.001$; right $>$ left, with local maximum corresponding to $[-28-18-18], t_{(17)}=3.78, p=0.001$ ). Hence, the interaction described above is significantly stronger in the right than the left hippocampus. 

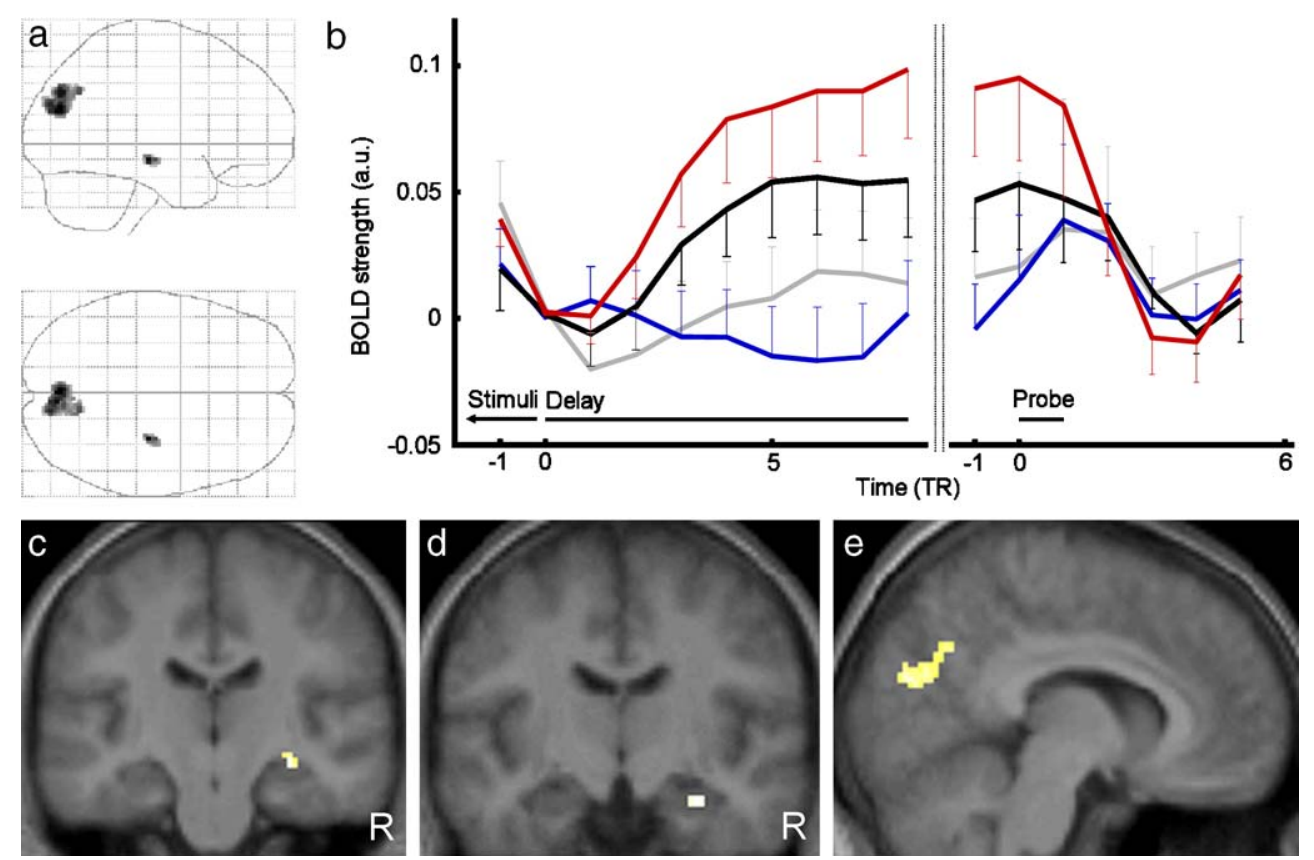

Fig. 3. Interaction between the factors ASSOCIATION and SPACE. Panel a shows an SPM (Intensity projection) of whole-brain activity; panel b shows averaged BOLD responses temporally aligned to stimulus offset and probe onset, separated for all conditions. Data are derived from the local maximum in the right hippocampus. Error bars represent standard deviations, and the black horizontal bars depict the averaged time window of the delay period, the stimulus, and the probe presentation (blue line 'location', black line 'color', red line 'location and number', gray line 'color and number'); panel c shows the right hippocampal interaction on a selected coronal slice of the averaged T1 image; panel d confirms the effect in the right hippocampus with a smaller smoothing kernel of 6 mm FWHM; and panel e shows the (pre)cuneus interaction on a selected sagittal slice.

We did not find a reliable main effect for the factors ASSOCIATION and SPACE in the hippocampus. To test whether these null effects are related to a thresholding artifact (type II error), we lowered the threshold to $p<0.01$ uncorrected. While we did not find a trend for a main effect of the factor ASSOCIATION, we did find one for the factor SPACE (cluster with local maximum at $\left.[26-12-22], t_{(51)}=2.41, p=0.01\right)$.

In addition to the hippocampal activation, at a cluster threshold of $p<0.05$ corrected for multiple comparisons over the whole brain, a caudomedial area at the dorsal border of BA18 (as indicated by the SPM anatomy toolbox (Eickhoff et al., 2005; Amunts et al., 2000)) covering parts of the cuneus and precuneus (cluster $p=0.003$ corrected) survived. This interaction is due to a larger difference in activation in the 'spatial single' vs. 'spatial multiple' comparison (with stronger responses for 'spatial multiple' than the 'spatial single' condition), as compared to the 'non-spatial single' vs. 'non-spatial multiple' (see Table 2, Fig. 3e).

Table 2

Regions showing delay-related activation on correct trials in the interaction between the factors ASSOCIATION and SPACE

\begin{tabular}{llrrrr}
\hline Interaction effects & Brodmann's area & $X$ & $Y$ & $Z$ & $t$ value \\
\hline (Pre)cuneus & $19 / 31$ & -2 & -76 & 24 & 4.21 \\
& $18 / 19$ & 6 & -78 & 22 & 4.07 \\
& $19 / 31$ & -2 & -76 & 34 & 4.06 \\
Right hippocampus & $7 / 31$ & 12 & -64 & 34 & 3.79 \\
\hline
\end{tabular}

\section{Discussion}

This study explored the circumstances under which sustained hippocampal activity is present during the delay period of a shortterm memory task. The results suggest that this hippocampal involvement is dependent on the information that needs to be maintained. Specifically, in our study, the right hippocampus is active during short-term memory maintenance of feature combinations that include spatial information (location-number associations), but not during maintenance of non-spatial feature combinations (color-number associations) or single items. The only other interaction found in this analysis was revealed in a caudomedial region including parts of the cuneus and the precuneus.

\section{Binding of trial features in the hippocampus}

Interestingly, our result suggests that the right hippocampus is specifically involved in the active maintenance of associations with spatial information, as compared to associative information in general. This finding is in line with previous studies conducted in rats (Gilbert and Kesner, 2002; Wood et al., 2004) and humans (Bohbot et al., 1998; Kessels et al., 2001), suggesting that hippocampal integrity is critically important for spatial, but not for non-spatial associations. However, this suggestion remains controversial following reports of involvement of the human hippocampus in associative binding beyond the spatial domain (e.g., Eichenbaum, 2004; Henke et al., 1999).

The reason for this potential specificity to spatial associative information might be the crucial role of the hippocampus in spatial processing. As initially found in rodents and later confirmed in primates and humans, the hippocampus has the ability to internally 
code spatial location by specific firing patterns (Ekstrom et al., 2003; O'Keefe and Dostrovsky, 1971). Hence, the hippocampus is well suited to bind together spatial information with the object information it receives (in)directly from the inferior temporal cortex (Mishkin, 1982). This binding is suggested to underlay the encoding and retrieval of episodic memories which invariably contain spatial information (O'Keefe and Nadel, 1978; Burgess et al., 2002). Consistent with this suggestion, a recent study in macaque monkeys identified hippocampal neurons that encode specific conjunctions of objects and their locations (Rolls et al., 2005). In humans, hippocampal activation has been shown in a short-term memory task when an object had to be bound to its spatial location (Mitchell et al., 2000a) and in studies that use real-world photographs of complex indoor and outdoor scenes depicting a large-scale spatial layout (Stern et al., 2001; Schon et al., 2004).

Alternatively, the dissociation found between spatial and non-spatial associative memory might not be based on the spatial feature per se, but on a difference in the demand for binding distributed neocortical codes. According to this interpretation, the hippocampus is involved in the integration of features that are not processed in an integrated fashion at earlier levels of processing. In the present setup, color and number may already be integrated in higher-order visual processing stages (Sakai and Miyashita, 1991), while the number and its location, two features which are processed in different cortical areas (Ungerleider and Mishkin, 1982; Andersen et al., 1985; Lueck et al., 1989), rely on the hippocampus for their integration. This interpretation would also be able to account for hippocampal activation in earlier studies on associative memory using nonsense objects, face/face pairs, or complex indoor/outdoor scenes (Vargha-Khadem et al., 1997; Schon et al., 2004; Ranganath et al., 2005a). Since the hippocampus receives input from the entire association cortex, it is positioned ideally for a region concerned with associating different aspects of an experience that have not been integrated at an earlier level of processing (Van Essen et al., 1992).

\section{Short-and long-term interactions and the medial temporal lobe}

Hippocampal involvement in short-term memory remains a topic of controversy. As described in the Introduction section, double dissociations between distinct types of memory have led to the definition of separate memory systems (Gabrieli, 1998). Patients with bilateral medial temporal lobe lesions typically exhibit normal short-term memory performance, but severely diminished consciously accessible long-term memory (i.e., episodic or declarative memory) (Scoville and Milner, 1957). Although the tasks used to investigate short-term memory in patients with bilateral medial temporal lobe lesions are of a wide variety using different kinds of stimuli, most of these investigations used only simple short-term memory tests such as span, delayed-match-tosample, or $n$-back tasks using single words, letters, or digits as stimuli (e.g., Scoville and Milner, 1957; Cave and Squire, 1992; Aggleton et al., 2000; Spiers et al., 2001; Ryan and Cohen, 2004). This leaves open the possibility that only short-term memory performance as assessed by these simple tests is unimpaired in amnesic patients. It is thus possible that the medial temporal lobe participates in more complex short-term memory tasks as suggested by our data and recent data by others (e.g., Schon et al., 2004; Ranganath et al., 2005a, Olson et al., 2006; for a review, see Ranganath and Blumenfeld, 2005).
To incorporate these findings, a number of recent models on memory formation emphasize the interaction between short- and long-term memory in the hippocampus (e.g., Atkinson and Shiffrin, 1968; Cowan, 1995; Baddeley, 2000). For example, Baddeley recently suggested an extension of the original working memory model (Baddeley and Hitch, 1974), accounting for some of the phenomena outlined above (Baddeley, 2000). According to the original model, working memory is a central cognitive capacity that supports several higher order cognitive functions and is composed of a central executive with two support systems, the phonological loop and the visuo-spatial sketchpad. Long-term knowledge can influence the operation of working memory, but the two are considered strictly separable cognitive systems (Baddeley and Logie, 1999). In the recent extension of the model, however, an episodic buffer was added (Baddeley, 2000). This new component plays an important role in feeding information into, and retrieving information from, episodic long-term memory. The episodic buffer shares some characteristics with the concept of episodic memory (Tulving, 1989) with respect to its principal mode of storing information in episodic format and its emphasis on integrative aspects but differs in that it is assumed to be a temporary store based on active maintenance. Thus, this episodic buffer might provide an interface between working and long-term memory. In emphasizing its short-term integrative role and the episodic format used, one may hypothesize that the episodic buffer is related to the prefrontal cortex, the medial temporal lobe, or both. The transient early role of the medial temporal lobe in long-term memory formation and sequence encoding in conjunction with the posterior parietal and prefrontal regions makes these areas likely candidates (cf. e.g., Eichenbaum, 2000; Fernández and Tendolkar, 2001; Simons and Spiers, 2003). In the extraction of information from working memory, a key function for the episodic buffer might be the integration of different subcomponents of working memory.

A second illustration of the re-evaluation of the dissociation between short- and long-term memory is the study conducted by Schon and colleagues. They showed that delay period activity in the hippocampus and related structures correlates positively with subsequent long-term memory performance (Schon et al., 2004; see also Ranganath et al., 2005a). This result is in line with a number of computational models that emphasize the necessity of active maintenance in medial temporal circuits for long-term memory formation (e.g., Jensen and Lisman, 1996, 2005; Koene et al., 2003). Thus, the activation of the hippocampus in this study may be due to the fact that the hippocampus is already actively forming long-term memory traces of the associations. This interpretation is supported by the study by Schon and colleagues, who show that medial temporal lobe involvement in working memory is a good predictor for successful long-term memory formation (Schon et al., 2004). The reason why we do not find this activation in the number-color association may be that this association is integrated in an earlier processing stage and thus does not need to be bound together for long-term memory storage. Hence, short- and long-term memory can interact seamlessly under certain circumstances, and the medial temporal lobe may serve as an interface where short-term and longterm memory interact in order to support (associative) memory capacities (Scoville and Milner, 1957; Olson et al., 2006). The current challenge is to further specify the exact conditions under which the hippocampus is involved in this interaction. Our study provides a possible framework for the integration of this emerging literature discussed above and the well-known role of the hippocampus in spatial and associative memory. 


\section{The (pre)cuneus}

The (pre)cuneus effect might be directly related to the effects observed in the hippocampus because this region is closely interlinked with the medial temporal lobe (Lavenex et al., 2002). The (pre)cuneus has been proposed to play an important role in episodic memory retrieval and thus in the process of retrieving information embedded in a spatial (and temporal) context (Wagner et al., 2005). Furthermore, a recent study investigating regions in which activity covaried with activity in the hippocampus identified a strong functional connectivity between the hippocampus and (among other regions) the precuneus and posterior cingulate gyrus during the early delay period of a working memory task with complex, trial unique stimuli (Ranganath et al., 2005b). This region and the hippocampus might therefore form a network of interacting brain areas mediating active maintenance of spatial associative information, and it might represent an interface between classical working memory areas in the prefrontal cortex and long-term memory areas in the medial temporal lobe (Kobayashi and Amaral, 2003).

\section{Conclusion}

This study characterizes specific circumstances under which the hippocampus is involved in a short-term memory task. We have shown that the right hippocampus is active during the delay interval of a short-term memory task specifically when associations between an object and its location have to be held online. These findings confirm and extend recent human lesion evidence (Olson et al., 2006) by showing that the spatial association held online specifically drives hippocampal contribution to short-term memory maintenance.

\section{Acknowledgments}

The authors thank Michael G.H. Coles for reading and commenting on an earlier draft of the manuscript, Lucia Talamini for inspiring discussion on the interpretation of the results, Paul Gaalman for technical support, Wim van de Riet for his help with anatomical labeling, Mark Rijpkema for providing a Matlab script for plotting the BOLD time courses, and the reviewers for helpful comments. This study was funded by a VENI grant from The Netherlands Organization for Fundamental Research (NWO, No. 451-02-037), awarded to RK.

\section{References}

Aggleton, J.P., McMackin, D., Carpenter, K., Hornak, J., Kapur, N., Halpin, S., Wiles, C.M., Kamel, H., Brennan, P., Carton, S., Gaffan, D., 2000. Differential cognitive effects of colloid cysts in the third ventricle that spare or compromise the fornix. Brain 123, 800-815.

Amunts, K., Malikovic, A., Mohlberg, H., Schormann, T., Zilles, K., 2000. Brodmann's area 17 and 18 brought into stereotaxic space-Where and how variable? NeuroImage 11, 66-84.

Atkinson, R.C., Shiffrin, R.M., 1968. Working memory: a proposed system and its control processes. In: Spence, K.W., Spence, J.T. (Eds.), The Psychology of Learning and Motivation: Advances in Research and Theory, vol. 2. Academic Press, New York, pp. 89-195.

Andersen, R., Essick, G., Siegel, R., 1985. Encoding of spatial location by posterior parietal neurons. Science 230, 456-458.

Baddeley, A., 2000. The episodic buffer: a new component of working memory? Trends Cogn. Sci. 4, 417-423.
Baddeley, A.D., Hitch, G.J., 1974. Working memory. In: Brower, G.H. (Ed.), The Psychology of Learning and Motivation: Advances in Research and Theory. Academic Press, New York, pp. 47-89.

Baddeley, A.D., Logie, R.H., 1999. Working memory: the multiplecomponent model. In: Miyake, A., Shah, P. (Eds.), Models of Working Memory. Mechanisms of Active Maintenance and Executive Control. Cambridge Univ. Press, Cambridge, pp. 28-61.

Bliss, T.V., Lomo, T., 1973. Long-lasting potentiation of synaptic transmission in the dentate area of the anaesthetized rabbit following stimulation of the perforant path. J. Physiol. 232, 331-356.

Bohbot, V.D., Kalina, M., Stepankova, K., Spackova, N., Petrides, M., Nadel, L., 1998. Spatial memory deficits in patients with lesions to the right hippocampus and to the right parahippocampal cortex. Neuropsychologia 36, 1217-1238.

Brasted, P.J., Bussey, T.J., Murray, E.A., Wise, S.P., 2003. Role of the hippocampal system in associative learning beyond the spatial domain. Brain 126, 1202-1223.

Burgess, N., Maguire, E.A., O'Keefe, J., 2002. The human hippocampus and spatial and episodic memory. Neuron 35, 625-641.

Cabeza, R., Dolcos, F., Graham, R., Nyberg, L., 2002. Similarities and differences in the neural correlates of episodic memory retrieval and working memory. Neurolmage 16, 317-330.

Cahusac, P.B.M., Miyashita, Y., Rolls, E.T., 1993. Response of hippocampal formation neurons in the monkey related to delayed spatial response and object-place memory tasks. Behav. Brain Res. 33, 229-240.

Cave, C.B., Squire, L.R., 1992. Intact verbal and non-verbal short-term memory following damage to the human hippocampus. Hippocampus 2, 151-163.

Chalfonte, B.L., Johnson, M.K., 1996. Feature memory and binding in young and older adults. Mem. Cognit. 244, 403-416.

Cowan, N., 1995. Attention and Memory: An Integrated Framework. Oxford Univ. Press, New York.

Dale, A.M., 1999. Optimal experimental design for event-related fMRI. Hum. Brain Mapp. 8, 109-114.

Davachi, L., Maril, A., Wagner, A.D., 2001. When keeping in mind supports later bringing to mind: neural markers of phonological rehearsal predict subsequent remembering. J. Cogn. Neurosci. 13, 1059-1070.

Duvernoy, H.M., Cabanis, E.A., Vannson, J.L., 1991. The Human Brain: Surface, Three-Dimensional Sectional Anatomy and MRI. SpringerVerlag, Wien.

Eichenbaum, H., 2000. A cortical-hippocampal system for declarative memory. Nat. Rev. Neurosci. 1, 41-50.

Eichenbaum, H., 2004. Hippocampus: cognitive processes and neural representations that underlie declarative memory. Neuron 44, $109-120$.

Eichenbaum, H., Otto, T., Cohen, N.J., 1994. Two functional components of the hippocampal memory system. Behav. Brain Sci. $17,449-517$.

Eickhoff, S.B., Stephan, K.E., Mohlberg, H., Grefkes, C., Fink, G.R., Amunts, K., Zilles, K., 2005. A new SPM toolbox for combining probabilistic cytoarchitectonic maps and functional imaging data. NeuroImage 25, 1325-1335.

Ekstrom, A.D., Kahana, M.J., Caplan, J.B., Fields, T.A., Isham, E.A., Newman, E.L., Fried, I., 2003. Cellular networks underlying human spatial navigation. Nature 425, 184-188.

Engert, F., Bonhoeffer, T., 1999. Dendritic spine changes associated with hippocampal long-term synaptic plasticity. Nature 399, 66-70.

Fernández, G., Tendolkar, I., 2001. Integrated brain activity in medial temporal and prefrontal areas predicts subsequent memory performance: human declarative memory formation at the system level. Brain Res. Bull. 55, 1-9.

Friston, K.J., Holmes, A.P, Poline, J.-B., Price, C.J., Frith, C.D., 1996. Detecting activations in PET and fMRI: levels of inference and power. NeuroImage 4, 223-235.

Friston, K.J., Asburner, J., Frith, C.D., Poline, J.B., Heather, J.D., Frackowiak, R.S., 1995a. Spatial registration and normalization of images. Hum. Brain Mapp. 3, 165-189. 
Friston, K.J, Holmes, A.P., Worsley, K., Poline, J-B., Frackowiak, R., 1995b. Statistical parametric maps in functional imaging: a general linear approach. Hum. Brain Mapp. 2, 189-210.

Fuster, J.M., Alexander, G.E., 1971. Neuron activity related to short-term memory. Science $173,652-654$.

Gabrieli, J.D.E., 1998. Cognitive neuroscience of human memory. Annu. Rev. Psychol. 49, 87-115.

Gilbert, P.E., Kesner, R.P., 2002. Role of the rodent hippocampus in pairedassociate learning involving associations between a stimulus and a spatial location. Behav. Neurosci. 116, 63-71.

Goldman-Rakic, P.S., 1987. Circuitry of primate prefrontal cortex and regulation of behaviour by representational memory. In: Plum, F., Mountcastle, V. (Eds.), Handbook of Physiology: The Nervous System. American Physiological Society, Bethesda, pp. 373-417.

Henke, K., Buck, A., Weber, B., Wieser, H.G., 1997. Human hippocampus establishes associations in memory. Hippocampus 7, 249-256.

Henke, K., Weber, B., Kneifel, S., Wieser, H.G., Buck, A., 1999. Human hippocampus associates information in memory. Proc. Natl. Acad. Sci. U. S. A. $96,5884-5889$.

Hopfinger, J.B., Buchel, C., Holmes, A.P., Friston, K.J., 2000. A study of analysis parameters that influence the sensitivity of event-related fMRI analyses. NeuroImage 11, 326-333.

Jensen, O., Lisman, J.E., 2005. Hippocampal sequence-encoding driven by a cortical multi-item working memory buffer. Trends Neurosci. 28, 67-72.

Jensen, O., Lisman, J.E., 1996. Novel lists of 7+-2 known items can be reliably stored in an oscillatory short-term memory network: interaction with long-term memory. Learn Mem. 3, 257-263.

Josephs, O., Turner, R., Friston, K.J., 1997. Event-related fMRI. Hum. Brain Mapp. 5, 243-248.

Kessels, R.P.C., De Haan, E.H.F., Kappelle, L.J., Postma, A., 2001. Varieties of human spatial memory: a meta-analysis on the effects of hippocampal lesions. Brain Res. Rev. 35, 295-303.

Kobayashi, Y., Amaral, D.G., 2003. Macaque monkey retrosplenial cortex: II. Cortical afferents. J. Comp. Neurol. 466, 48-79.

Koene, R.A., Gorchetchnikov, A., Cannon, R.C., Hasselmo, M.E., 2003. Modeling goal-directed spatial navigation in the rat based on physiological data from the hippocampal formation. Neural Netw. 16, 577-584.

Lavenex, P., Suzuki, W.A., Amaral, D.G., 2002. Perirhinal and parahippocampal cortices of the macaque monkey: projections to the neocortex. J. Comp. Neurol. 447, 394-420.

Lueck, C.J., Zeki, S., Friston, K.J., Deiber, M.P., Cope, P., Cunningham, V. J., Lammertsma, A.A., Kennard, C., Frackowiak, R.S., 1989. The colour centre in the cerebral cortex of man. Nature 340, 386-389.

Luo, J., Niki, K., 2005. Does hippocampus associate discontiguous events? Evidence from event-related fMRI. Hippocampus 15, 141-148.

Maldjian, J.A., Laurienti, P.J., Kraft, R.A., Burdette, J.H., 2003. An automated method for neuroanatomic and cytoarchitectonic atlas-based interrogation of fMRI data sets. NeuroImage 19, 1233-1239.

Miller, E.K., Li, L., Desimone, R., 1993. Activity of neurons in anterior inferior temporal cortex during a short-term memory task. J. Neurosci. 13, 1460-1478.

Miller, E.K., Erickson, C.A., Desimone, R., 1996. Neural mechanisms of visual working memory in prefrontal cortex of the macaque. J. Neurosci. $16,5154-5167$.

Mitchell, K.J., Johnson, M.K., Raye, C.L., D’Esposito, M., 2000a. fMRI evidence of age-related hippocampal dysfunction in feature binding in working memory. Cogn. Brain Res. 10, 197-206.

Mitchell, K.J., Raye, C.L., Mather, M., D’Esposito, M., 2000b. Aging and reflective processes of working memory: binding and test load deficits. Psychol. Aging 15, 527-541.

Mishkin, M., 1982. A memory system in the monkey. Trans R. Soc. Lond. B. Biol. Sci. 298, 85-95.

Miyashita, Y., Chang, H.S., 1998. Neuronal correlate of pictorial short-term memory in the primate temporal cortex. Nature 331, 68-70.

O'Keefe, J., Dostrovsky, J., 1971. The hippocampus as a spatial map. Preliminary evidence from unit activity in the freely-moving rat. Brain Res. 34, 171-175.
O’Keefe, J., Nadel, L., 1978. The Hippocampus as a Cognitive Map. Oxford Univ. Press, Oxford.

Olson, I.R., Page, K., Sledge Moore, K., Chatterjee, A., Verfaellie, M., 2006. Working memory for conjunctions relies on the medial temporal lobe. J. Neurosci. 26, 4596-4601.

Ranganath, C., Blumenfeld, R.S., 2005. Doubts about double dissociations between short- and long-term memory. Trends Cogn. Sci. 9, 374-380.

Ranganath, C., D'Esposito, M., 2001. Medial temporal lobe activity associated with active maintenance of novel information. Neuron 31, 865-873.

Ranganath, C., Cohen, M.X., Brozinsky, C., 2005a. Working memory maintenance contributes to long-term memory formation: neural and behavioral evidence. J. Cogn. Neurosci. 17, 994-1010.

Ranganath, C., Heller, A., Cohen, M.X., Brozinsky, C.J., Rissman, J., 2005b. Functional connectivity with the hippocampus during successful memory formation. Hippocampus 15, 997-1005.

Rolls, E.T., Xiang, J., Franco, L., 2005. Object, space, and object-space representation in the primate hippocampus. J. Neurophysiol. 94, 833-844.

Ryan, J.D., Cohen, N.J., 2004. Processing and short-term retention of relational information in amnesia. Neuropsychologia 42, 497-511.

Sakai, K., Miyashita, Y., 1991. Neural organization for the long-term memory of paired association. Nature 354, 152-155.

Schacter, D.L., Tulving, E., 1994. What are the memory systems of 1994 ? In: Tulving, E., Schacter, D.L. (Eds.), Memory Systems. MIT Press, Cambridge, pp. 1-38.

Schon, K., Hasselmo, M.E., LoPresti, M.L., Tricarico, M.D., Stern, C.E., 2004. Persistence of parahippocampal representation in the absence of stimulus input enhances long-term encoding: a functional magnetic resonance imaging study of subsequent memory after a delayed matchto-sample task. J. Neurosci. 24, 11088-11097.

Scoville, W.B., Milner, B., 1957. Loss of recent memory after bilateral hippocampal lesions. J. Neurol., Neurosurg. Psychiatry 20, $11-21$.

Shallice, T., Warrington, E.K., 1970. Independent functioning of verbal memory stores: a neuropsychological study. Q. J. Exp. Psychol. 22, 261-273.

Shors, T.J., Miesegaes, G., Beylin, A., Zhao, M., Rydel, T., Gould, E., 2001. Neurogenesis in the adult is involved in the formation of trace memories. Nature 410, 372-376.

Simons, J.S., Spiers, H.J., 2003. Prefrontal and medial temporal lobe interactions in long-term memory. Nat. Rev., Neurosci. 4, 637-648.

Spiers, H.J., Maguire, E.A., Burgess, N., 2001. Hippocampal amnesia. Neurocase 7, 357-382.

Squire, L.R., Knowlton, B., Musen, G., 1993. The structure and organization of memory. Annu. Rev. Psychol. 44, 453-495.

Stern, C.E., Sherman, S.J., Kirchhoff, B.A., Hasselmo, M.E., 2001. Medial temporal and prefrontal contributions to working memory tasks with novel and familiar stimuli. Hippocampus 11, 337-346.

Sternberg, S., 1966. High-speed scanning in human memory. Science 153, 652-654.

Suzuki, W.A., Miller, E.K., Desimone, R., 1997. Object and place memory in the macaque entorhinal cortex. J. Neurophysiol. 78, 1062-1081.

Toni, I., Schluter, N.D., Josephs, O., Friston, K.J., Passingham, R.E., 1999. Signal-, set- and movement-related activity in the human brain: an eventrelated fMRI study. Cereb. Cortex 9, 35-49.

Tulving, E., 1989. Memory: performance, knowledge and experience. Eur. J. Cogn. Psychol. 1, 3-26.

Ungerleider, L.G., Mishkin, M., 1982. Two cortical visual systems. In: Ingle, D.J., Goodale, M.A., Mansfield, R.J.W. (Eds.), Analysis of Visual Behavior. MIT Press, Cambridge, pp. 549-586.

Vallar, G., Papagno, C., 1995. Neuropsychological impairments of shortterm memory. In: Baddeley, A.D., Wilson, B.A., Watts, F.N. (Eds.), Handbook of Memory Disorders. Wiley, New York.

Van Essen, D., Anderson, C., Felleman, D., 1992. Information processing in the primate visual system: an integrated systems perspective. Science $255,419-423$. 
Vargha-Khadem, F., Gadian, D.G., Watkins, K.E., Connelly, A., Van Paesschen, W., Mishkin, M., 1997. Differential effects of early hippocampal pathology on episodic and semantic memory. Science 277, 276-380.

Wager, T.D., Smith, E.E., 2003. Neuroimaging studies of working memory: a meta-analysis. Cogn. Affect. Behav. Neurosci. 3, 255-274.

Wagner, A.D., 1999. Working memory contributes to human learning and remembering. Neuron 22, 19-22.
Wagner, A.D., Shannon, B.J., Kahn, I., Buckner, R.L., 2005. Parietal lobe contributions to episodic memory retrieval. Trends Cogn. Sci. 9, $445-453$.

Watanabe, T., Niki, H., 1985. Hippocampal unit activity and delayed response in the monkey. Brain Res. 325, 241-254.

Wood, E.R., Agster, K.M., Eichenbaum, H., 2004. One-trial odor-reward associations: a form of event memory not dependent on hippocampal function. Behav. Neurosci. 118, 526-539. 